\title{
Complications des Mastites Puerpérales: Aspects Cliniques et Thérapeutiques dans un Hôpital de Zone Rurale au Togo
}

\author{
Biréga Koutora \\ Service de Chirurgie, \\ Hôpital de l'Ordre de Malte d'Elavagnon, Togo \\ Edem Têtê Kouevi-Koko \\ Service de Brûlés, Plaies et Cicatrisation, \\ CHU Sylvanus Olympio - Lomé, Togo \\ Mibirim Agbogawo \\ Clinique Médico-Chirurgicale (Pavillon Militaire), \\ CHU Sylvanus Olympio - Lomé, Togo \\ Kodjo Massogblé Nagbé Koffi \\ Déladem Yaovi Guinhouya \\ Service de Gynécologie - Obstétrique, \\ CHU Sylvanus Olympio - Lomé, Togo \\ Kokou Kouliwa Kanassoua \\ Département de Chirurgie et Spécialités Chirurgicales, \\ Faculté des Sciences de la Santé, Université de Lomé, Togo
}

\section{Doi:10.19044/esj.2021.v17n34p86}

Submitted: 29 July 2021

Accepted: 13 September 2021

Published: 30 September 2021
Copyright 2021 Author(s)

Under Creative Commons BY-NC-ND 4.0 OPEN ACCESS

Cite As:

Koutora B., Kouevi-Koko E.T., Agbogawo M., Nagbé Koffi K.M., Guinhouya D.Y. \& Kanassoua K.K. (2021). Complications des Mastites Puerpérales : Aspects Cliniques et Thérapeutiques dans un Hôpital de Zone Rurale au Togo. European Scientific Journal, ESJ, 17(34), 86.

https://doi.org/10.19044/esj.2021.v17n34p86

\section{Résumé}

Introduction: L’objectif de cette étude était de décrire les aspects cliniques et thérapeutiques des complications des mastites puerpérales dans un hôpital confessionnel en zone rurale au Togo. Méthodologie: Il s'est agi d'une étude transversale descriptive menée à l'Hôpital de l'Ordre de Malte d'Elavagnon de Janvier à Décembre 2020. Etaient incluses, les patientes allaitantes prises en charge pour cellulites, abcès et fasciites nécrosantes du sein. Résultats: 47 patientes ont été enregistrées avec un âge moyen de 24,5 
$\pm 5,1$ ans. Il s'agissait majoritairement d'agricultrices (61,7\%). Toutes les patientes avaient eu recours au traitement indigène avant leur admission à l'hôpital. Les lésions étaient représentées par 27 cas d'abcès (57,4\%), 14 cas de fasciite nécrosante (29,8\%), et 6 cas de cellulite (12,8\%). Le sein droit était le plus atteint $(72,3 \%)$. La prise en charge comportait un traitement médical fait d'analgésie et d'antibiothérapie probabiliste, et un traitement chirurgical qui était fonction des lésions. L'évolution était bonne dans tous les cas. Conclusion: La mastite puerpérale est une affection handicapante de la femme allaitante. Le retard à la prise en charge et le recours à des traitements inadaptés exposent à des complications plus graves dont la prise en charge est plus longue.

Mots clés: Mastite Puerpérale, Abcès, Cellulite, Fasciite Nécrosante, Zone Rurale

\title{
Complications of Puerperal Mastitis: Clinical and Therapeutic Aspects in a Rural Zone Hospital in Togo
}

\author{
Biréga Koutora \\ Service de Chirurgie, \\ Hôpital de l'Ordre de Malte d'Elavagnon, Togo \\ Edem Têtê Kouevi-Koko \\ Service de Brûlés, Plaies et Cicatrisation, \\ CHU Sylvanus Olympio - Lomé, Togo \\ Mibirim Agbogawo \\ Clinique Médico-Chirurgicale (Pavillon Militaire), \\ CHU Sylvanus Olympio - Lomé, Togo \\ Kodjo Massogblé Nagbé Koffi \\ Déladem Yaovi Guinhouya \\ Service de Gynécologie - Obstétrique, \\ CHU Sylvanus Olympio - Lomé, Togo

\section{Kokou Kouliwa Kanassoua}

Département de Chirurgie et Spécialités Chirurgicales, Faculté des Sciences de la Santé, Université de Lomé, Togo

\begin{abstract}
Introduction: The aim of this study was to describe clinical and therapeutic aspects of puerperal mastitis complications in a confessional hospital in rural zone in Togo. Methodology: It was a descriptive transversal study carried out at " Ordre de Malte » Hospital of Elavagnon, including breastfeeding patients treated for cellulitis, abcess, and necrotizing fasciitis of the breast, from January to December, 2020. Results: 47 patients were
\end{abstract}


registered with an average age of $24.5 \pm 5.1$ years old. The patients were mainly farmers (61.7\%). All patients undergone indigenous treatment before admission to hospital. The lesions were abcesses in 27 cases (57.4\%), necrotizing fasciitis in 14 cases (29.8\%), and cellulitis in 6 cases (12.8\%). Management included treatment with analgesics and probabilistic antibiotics, and surgical treatment. The outcome was good il all cases. Conclusion: Puerperal mastitis is a disabling condition of breastfeeding women. The delay in treatment and the use of inappropriate treatments expose to complications that take a long time to take care of.

Keywords: Puerperal Mastitis, Abcess, Cellulitis, Necrotizing Fasciitis, Rural Zone

\section{Introduction}

La mastite puerpérale aussi appelée mastite lactationnelle est une inflammation du sein souvent associée à une infection, survenant pendant la période d'allaitement. Elle touche 10 à $20 \%$ des femmes pendant les six premiers mois de lactation, les six à huit premières semaines étant les plus à risque (Mertz, 2014 ; Wilson, 2020). Son diagnostic est essentiellement clinique, basé sur l'apparition d'une douleur, d'une rougeur, d'une chaleur et d'une tuméfaction du sein parfois accompagnée d'un état fébrile. Le retard à la prise en charge et le recours à des traitements inadaptés exposent à des complications comme l'abcès, la cellulite et la fasciite nécrosante du sein (Mertz, 2014 ; Yu, 2018). En milieu rural, en raison des difficultés d'accès aux soins de santé, mais aussi des croyances des populations, le recours au traitement traditionnel est quasi-systématique devant toute affection. La consultation dans les structures conventionnelles de soins se fait alors souvent après échec du traitement indigène au stade des complications. Ainsi, les patientes souffrant de mastite puerpérale sont vues à l'hôpital au stade de complications, après des soins reçus des charlatans et tradi-praticiens. Cette étude a pour but de décrire les aspects cliniques et thérapeutiques des complications des mastites puerpérales prises en charge à l'Hôpital de l'Ordre de Malte d'Elavagnon, un hôpital confessionnel situé en zone rurale au Togo.

\section{Méthodologie}

Il s’est agi d'une étude transversale descriptive menée à l'Hôpital de l'Ordre de Malte d’Elavagnon de Janvier à Décembre 2020 (un an). Etaient incluses, toutes les patientes allaitantes prises en charge dans ledit hôpital pour abcès, cellulites et fasciites nécrosantes du sein. Les patientes étaient recrutées au fur et à mesure de leur admission à l'hôpital à partir d'une fiche d'enquête. Le diagnostic était clinique. Le traitement comportait un volet médical, un volet chirurgical, et des mesures générales. Le volet médical était constitué 
d'un traitement antalgique et d'un traitement antibiotique probabiliste fait d'Amoxicilline-acide clavulanique ou d'Erythromycine en cas de cellulite ou d'abcès, et d'Amoxicilline-acide clavulanique associé au Métronidazole lorsqu'il s'agissait d'une fasciite nécrosante. Aucun examen cytobactériologique n'était fait car non réalisable localement. Le traitement chirurgical était fonction de la lésion et consistait en incision - drainage d'abcès ou de sérosités, décapage suivi de greffe de peau à distance du processus infectieux. Les mesures générales comportaient essentiellement l'expression manuelle du lait au niveau du sein.

Les paramètres étudiés étaient: l'âge, la profession, les aspects lésionnels et les modalités thérapeutiques. Le traitement des données a été fait à partir du tableur Excel 2019. Les images ont été traitées sur le logiciel Paint.

\section{Résultats}

\section{Aspects épidémiologiques}

Quarante - et - sept patientes ont été enregistrées avec un âge moyen de 24,5 \pm 5,1 ans (extrêmes : 17 ans et 36 ans). Il s'agissait majoritairement d'agricultrices (61,7\%). La parité moyenne était de 1,8 (extrêmes 1 et 5). Les paramètres épidémiologiques sont récapitulés dans le tableau I.

Tableau I. Récapitulatif des paramètres épidémiologiques des complications des mastites puerpérales à l'Hôpital de l'Ordre de Malte d'Elavagnon

\begin{tabular}{|c|c|c|c|}
\hline & & Effectif & Pourcentage \\
\hline Tranche d'âge & {$[17-25[$} & 26 & 55,3 \\
\hline & {$[25-35[$} & 19 & 40,4 \\
\hline & $\geq 35$ & 2 & 4,3 \\
\hline Profession & Agricultrices & 29 & 61,7 \\
\hline & Coiffeuses & 12 & 25,5 \\
\hline & Elèves & 3 & 6,4 \\
\hline & Tissérantes & 3 & 6,4 \\
\hline Parité & 1 & 19 & 40,4 \\
\hline & 2 & 18 & 38,3 \\
\hline & 3 & 7 & 14,9 \\
\hline & 4 & 2 & 4,3 \\
\hline & 5 & 1 & 2,1 \\
\hline
\end{tabular}

\section{Aspects cliniques}

Le délai d'apparition des symptômes variait entre 23 jours et 8 mois d'allaitement. Toutes les patientes avaient eu recours au traitement à l'indigénat avant leur admission à l'hôpital. Trois patientes (6,4\%) étaient en arrêt d'allaitement à cause du décès de leurs bébés. Les lésions étaient dominées par les abcès ( 27 cas / 57,4\%). On avait 14 cas de fasciite nécrosante (29,8\%) et 6 cas de cellulite (12,8\%). Les figures 1, 2, et 3 sont des exemples illustratifs d'images respectivement d'abcès, de cellulite et de fasciite 
nécrosante du sein retrouvés dans cette série. Le sein droit était le plus atteint (34 cas / 72,3\%). Il n’y avait aucun cas où les deux seins étaient atteints.

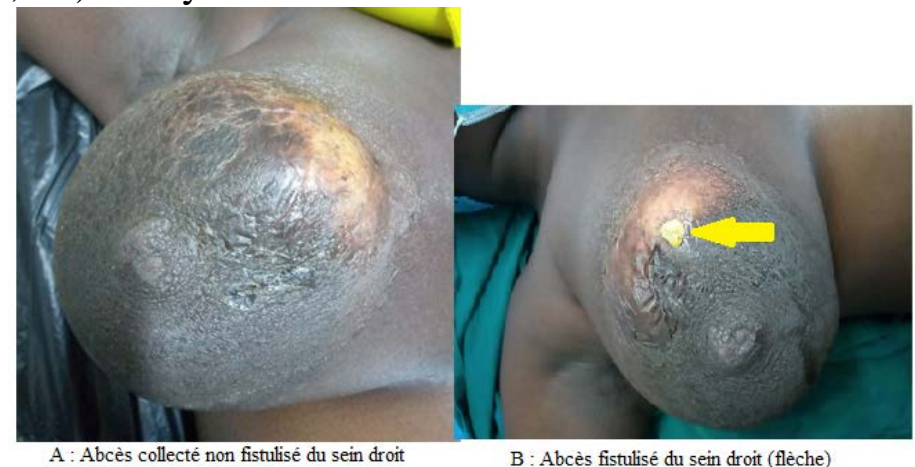

Figure 1. Images d'abcès du sein

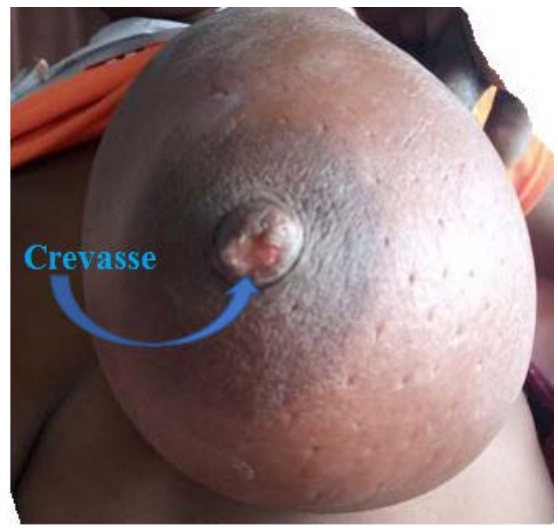

Figure 2. Cellulite du sein gauche avec crevasse au mamelon
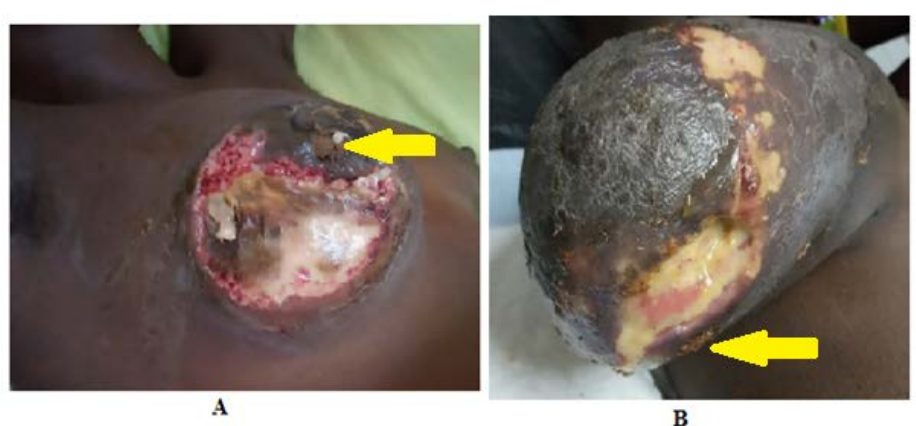

Figure 3. Fasciite nécrosante du sein droit avec dépôt de produits de décoction (flèche). A : patiente de 33 ans, quatrième geste - quatrième pare. $\mathrm{B}$ : Patiente de 19 ans, primipare

\section{Aspects thérapeutiques}

Le traitement médical comportait la prise en charge de la douleur et le traitement antibiotique probabiliste. Le traitement de la douleur était fait à base d'Acide Acétylsalicylique lorsqu'il n’y avait plus d'allaitement (à cause du 
décès du bébé), et de Paracétamol dans les autres cas avec poursuite de l'allaitement maternel. L'antibiothérapie probabiliste était à base d'Amoxicilline-acide clavulanique et d'Erythromycine respectivement dans 29 cas $(61,7 \%)$ et 4 cas $(8,5 \%)$ en cas de cellulite ou d'abcès ; et d'Amoxicilline-acide clavulanique associé au Métronidazole lorsqu'il s'agissait d'une fasciite nécrosante (14 cas / 29,8\%). La durée médiane de l'antibiothérapie était de 8 jours (extrêmes : 7 jours et 18 jours).

Le traitement chirurgical était fonction des lésions. Les abcès étaient traités par incision - drainage de pus. Une ponction à l'aveugle faite dans deux cas d'abcès s'était soldée par une récidive respectivement au troisième jour et cinquième jour, imposant une incision. Les cellulites étaient traitées par incision et expression des sérosités. La prise en charge chirurgicale des fasciite nécrosantes consistait en des décapages et soins locaux réguliers. Une greffe de peau autologue était réalisée dans 12 cas (25,5\%) à distance du processus infectieux. Le délai médian pour la réalisation de la greffe de peau était de 21,3 jours (15 jours et 29 jours). La cicatrisation spontanée était obtenue dans 2 cas de fasciites nécrosantes après un délai moyen de 71,3 jours ; et dans les plaies d'incisions d'abcès et de cellulites après un délai moyen respectivement de 25,7 jours, et 17,2 jours. La figure 4 est un exemple illustratif d'évolution d'une fasciite nécrosante du sein prise en charge dans cette série.

Les autres mesures thérapeutiques étaient essentiellement constituées de l'expression du lait de façon manuelle au niveau du sein malade, et de la poursuite de l'allaitement maternel par le sein sain.

L'évolution était bonne dans tous les cas. Le recul moyen de suivi était de 6,3 mois. La mortalité était nulle.

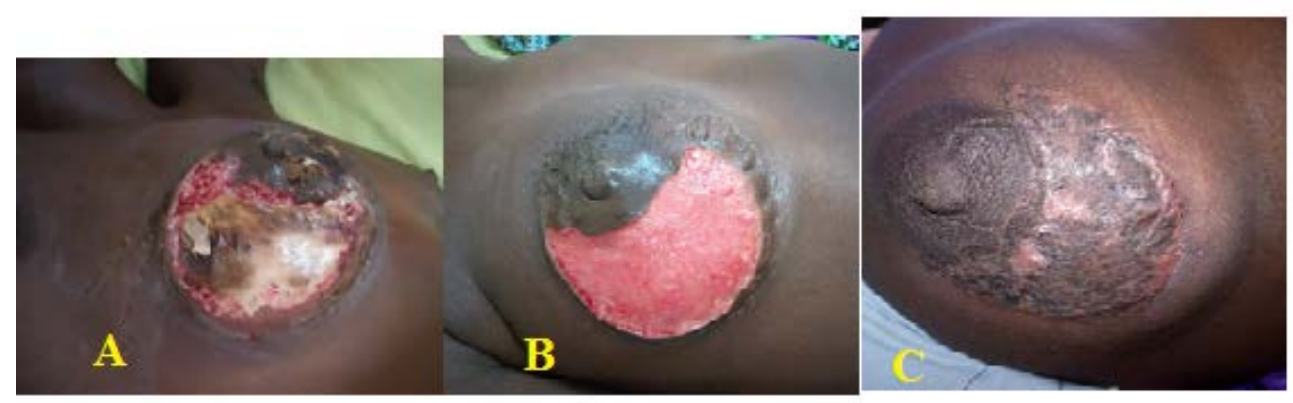

Figure 4. Images illustratives d'évolution d'une fasciite nécrosante du sein sous prise en

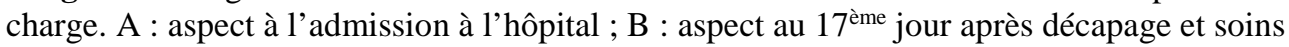

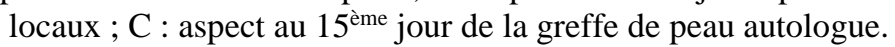

\section{Discussion}

La mastite puerpérale est une morbidité maternelle qui affecte le bienêtre des mères et de leurs bébés. Elle résulte souvent d'une stase laiteuse, conséquence d'une vidange incomplète du lait. La stase laiteuse peut évoluer 
vers une mastite non infectieuse, laquelle devient infectieuse suite à l'entrée bactérienne rétrograde à travers les canaux galactophores ou le mamelon traumatisé, mais aussi par voie hématogène ou lymphatique (Abou-Dakn, 2010 ; Yu, 2018). La vidange incomplète du lait est en général le résultat de techniques pauvres d'allaitement, ou de l'inexpérience de l'allaitement (Khanal, 2015 ; Yu, 2018). En effet, dans cette série, 55\% des patientes avaient moins de 25 ans, et $40 \%$ étaient des primipares. Cependant, la mastite puerpérale peut affecter toute femme allaitante quels que soient l'âge et la parité (Bond, 2017).

Débutée tôt, la prise en charge de la mastite puerpérale est simple et repose sur la vidange du lait, le traitement de la douleur et éventuellement un traitement antibiotique. Cette prise en charge rapide et adaptée évite l'évolution vers des complications (Jahanfar, 2016 ; Yu, 2018). Ces dernières étaient les motifs de consultation dans cette étude, en raison du retard à la consultation et du recours systématique au traitement indigène. Le retard de prise en charge à ce stade de complications peut être fatal notamment en cas d'abcès ou de fasciite nécrosante dont le risque immédiat est le choc septique. Le diagnostic dans cette série était clinique. Aucune culture n'était faite pour l'identification des germes en cause, du fait de l'insuffisance du plateau technique local. Le traitement antibiotique était probabiliste en se basant sur les données de la littérature concernant les mastites et leurs complications. En effet, les germes en cause peuvent être les bactéries présentes sur la peau ou les bactéries pathogènes. Il s'agit souvent de Staphylococcus aureus, de Streptocoques du groupe B, et des anaérobies; mais aussi parfois de: Coagulase Negative Staphylococci, Viridans Streptococci, Enterococcus feacalis (Foxman, 2002; Bond, 2017; Yu, 2018; Hajji, 2020). L'antibiothérapie probabiliste instituée dans cette étude a été conduite jusqu'à la fin du traitement avec un bon résultat clinique. Le traitement chirurgical qui s'y associait à ce stade de complications rendait la prise en charge plus couteuse et plus longue pour la patiente et son entourage, avec le risque de préjudice esthétique. Ce qui souligne l'intérêt de la précocité de consultation dans les structures de santé conventionnelles, afin qu'un traitement adéquat puisse y être débuté et éviter la survenue des complications. Ainsi, des activités de sensibilisations devraient être menées auprès des populations des zones rurales mais aussi des charlatans et tradi-praticiens, les incitant à se rendre précocement à l'hôpital pour se faire consulter, et à éviter le recours systématique au traitement indigène devant toute affection. Par ailleurs, les prestataires de santé en charge de l'accouchement doivent apprendre aux mères la vidange complète du lait et l'hygiène afin de prévenir la mastite ; et les sensibiliser sur la prise en charge des complications de l'allaitement. 


\section{Conclusion}

La mastite puerpérale est une complication de l'allaitement dont la prévention est essentielle. Le retard à la consultation et le recours à des traitements inadaptés exposent à des complications. Ces complications pourraient être évitées par des activités de sensibilisation incitant les populations à se faire consulter précocement à l'hôpital et à éviter le recours systématique au traitement indigène devant toute affection. La prise en charge adéquate des complications des mastites puerpérales quoique plus couteuse et longue que celle de la mastite simple donne des résultats satisfaisants.

\section{References:}

1. Abou-Dakn, M., Richardt, A., Schaefer-Graf, U., \& Wöckel, A. (2010). Inflammatory breast diseases during lactation : milk stasis, puerperal mastitis, abscesses of the breast, and malignant tumors current and evidence-based strategies for diagnosis and therapy. Breast Care (Basel) ; 5(1) : 33-7

2. Bond, DM., Morris, JM., \& Nassar, N. (2017). Study protocol : evaluation of the probiotic Lactobacillus Fermentum CECT5716 for the prevention of mastitis in breastfeeding women : a randomised controlled trial. BMC Pregnancy and Childbirth; $17: 148$ doi 10.1186/s12884-017-1330-8

3. Foxman, B., D’Arcy, H., Gillespie, B., Bobo, JK., \& Schwartz, K. (2002). Lactation mastitis: Occurrence and medical management among 946 breastfeeding women in the United States. Am J Epidemiol ; 155 : 103-14

4. Hajji, A., Toumi, D., Njima, M., Cherif, O., Ben Salem, A., Korbi, A., \& Faleh, R. (2020). Fasciite nécrosante du sein: traitement conservateur et préjudice esthétique, rapport d'un cas. Pan Afr Med J Clinical Med ; 3(188) doi 10.11604/pamj-cm.2020.3.188.23858

5. Jahanfar, S., Ng, CJ., \& Teng, CL. (2016). Antibiotics for mastitis in breastfeeding women. Sao Paulo Med J ; 134(3) : 273

6. Khanal, V., Scott, JA., Lee, AH., \& Binns, CW. (2015). Incidence of Mastitis in the Neonatal Period in a Traditional Breastfeeding Society : Results of a Cohort Study. Breastfeeding Med ; 10(10) : 481-7

7. Mertz, L., De Courten, C., \& Orasch, C. (2014). Infections du sein. Rev Med Suisse ; 10 : 925-30

8. Wilson, E., Woodd, SL., \& Benova, L. (2020). Incidence of and Risk Factors for Lactational Mastitis : A Systematic Review. J of Human Lactation ; 36(4) : 673-86

9. Yu, Z., Sun, S., \& Zhang Y. (2018). High-Risk Factors for Suppurative Mastitis in Lactating Women. Med Sci Monit ; 24 : 4192-7 